\title{
Inclusión financiera en Colombia. Análisis de su evolución en términos de eficiencia
} relativa* $^{*}$

\author{
Financial inclusion in Colombia. Analysis of its evolution in terms of relative efficiency \\ Inclusão financeira na Colômbia. Análise da sua evolução em termos de eficiência relativa
}

Gloria Isabel Rodriguez-Lozano a

DOI: https://doi.org/10.11144/Javeriana.cc18-45.ifca

Universidad Nacional de Colombia, Colombia

girodriguezl@unal.edu.co

ORCID: http://orcid.org/0000-0001-6906-9223

Fecha de recepción: 19 Septiembre 2016 Fecha de aprobación: 15 Marzo 2017

\section{Resumen:}

Esta investigación analiza la situación y evolución de la inclusión financiera en Colombia desde un punto de vista diferente: desde la eficiencia relativa, que maneja las entidades financieras como objeto específico — no global— de análisis. Por esto y por la metodología usada, esta investigación es pionera. La metodología que se utiliza es el Análisis Envolvente de Datos (Data Envelopment Analysis, DEA), metodología de programación lineal avanzada que mediante un doble proceso de optimización proporciona un único índice de eficiencia relativa para cada una de las unidades estudiadas, con la fortaleza de analizar las variables en un escenario de utilización de multirrecursos y de obtención de multisalidas. Da respuesta a los siguientes interrogantes: ¿Qué tipo de entidades es eficiente y en qué número para cada año? ¿Qué resultados se obtienen cuando se compara el comportamiento, en términos de eficiencia relativa, de los años 2013 y 2014? Los resultados, en la comparación, muestran que globalmente hay un desmejoramiento en el comportamiento de la eficiencia relativa de las instituciones evaluadas: disminuyen las unidades con altos índices y aumentan las unidades con bajos índices.

Códigos JEL: C14, C67, G20

Palabras clave: Análisis envolvente de datos, (Data Envelopment Analysis, DEA), inclusión financiera, eficiencia de la inclusión financiera.

\section{Abstract:}

This research analyzes the situation and evolution of financial inclusion in Colombia from a different point of view: from that of relative efficiency, which manages the financial entities as a specific -not global- object of analysis. Given this point of view and the methodology used, this is a pioneering research. The methodology used is Data Envelopment Analysis (DEA), an advanced linear programming methodology that provides a single index of relative efficiency for each of the units studied through a double optimization process, with the advantage of analyzing the variables in a scenario of multi-resource use and obtaining multiple outputs. It answers the following questions: what kind of entity is efficient and in what amount for each year? What results are obtained when comparing the behavior, in terms of relative efficiency, of the years 2013 and 2014? The results in the comparison show that globally there is deterioration in the behavior of the relative efficiency of the evaluated institutions, that is, units with high indexes decrease and units with low indexes increase.

Keywords: Data envelopment analysis, DEA, financial inclusion, efficiency of financial inclusion.

\section{Resumo:}

Esta pesquisa analisa a situação e evolução da inclusão financeira na Colômbia desde um ponto de vista diferente: desde a eficiência relativa, que maneia as entidades financeiras como objeto específico — não global— de análise. Por isso e pela metodologia usada, esta pesquisa é pioneira. A metodologia utilizada é a Análise de Envolvimento de Dados (Data Envelopment Analysis, DEA), metodologia de programação linear avançada que mediante um processo duplo de optimização proporciona um único índice de eficiência relativa para cada uma das unidades estudadas, com a fortaleza de analisar as variáveis em um panorama de utilização de multirrecursos e de obtenção de multisalidas. Da resposta às seguintes perguntas: Que tipo de entidades é eficiente e em qual número para cada ano? Quais os resultados obtidos quando comparado o comportamento, em termos de eficiência relativa, nos anos 2013 e 2014? Os resultados, na comparação, mostram que globalmente têm deterioração do comportamento da eficiência relativa das instituições avaliadas: diminuem as unidades com altos índices e aumentam as unidades com baixos índices.

Notas de autor 
Palavras-chave: Análise de Envolvimento de dados, (Data Envelopment Analysis, DEA), inclusão financeira, eficiência da inclusão financeira.

\section{Introducción}

El objetivo principal de la estrategia mundial de desarrollo tiene qué ver con el bienestar de las personas, mediante la erradicación de la pobreza extrema y el hambre. Una de estas estrategias es el logro de los Objetivos de Desarrollo del Milenio. Tanto es así que la principal preocupación en la agenda de desarrollo ha sido la reducción de la vulnerabilidad humana y la potenciación de las personas; para Amartya Sen (1999), premio Nobel de Economía de 1998, el desarrollo significa aumentar la capacidad de elegir de las personas entre diferentes alternativas. La inclusión financiera se ha considerado como uno de los caminos por los cuales esta capacidad puede ser mejorada; tener acceso a financiación es un importante requisito para el crecimiento económico, ya que conduce a una mayor inversión, a un aumento en el ingreso y a una mayor autonomía de los hogares, al liberarlos de los círculos de pobreza (Solo, 2008). Adicionalmente, para Cecilia Vera y Daniel Titelman (2013, p. 9), "Un requisito para que los sistemas financieros sean funcionales a un desarrollo económico y social sostenible es el de la inclusividad". Para la Comisión Multisectorial de Inclusión Financiera, CMIF (2015, p. 5): "Las políticas de inclusión financiera son herramientas promotoras de la inclusión social y el desarrollo económico”.

Esta misma idea se expresa en el Global Financial Development Report (2013, p. 26):

En los sistemas financieros no inclusivos son las empresas pequeñas y los individuos de menores ingresos quienes no logran acceder a servicios financieros. Esto a su vez se constituye en un mecanismo que refuerza las desigualdades, ya que estos agentes se apoyan exclusivamente en recursos propios para todo, desde su propia educación hasta poner un negocio, o invertir en una oportunidad que consideran ventajosa.

El programa de inclusión financiera ha sido identificado como una importante estrategia para reducir la pobreza y la vulnerabilidad, mediante la inclusión de personas de todas las condiciones en el sistema de la banca tradicional; pues de esta manera se eleva la capacidad de inversión y se desarrollan habilidades de ahorro y empresariales que conducen a mayores ingresos y, en consecuencia, a una mejor vida (Chakrabarty, 2009). Tanto es así que en 2006, el United Nations Committee on Building Inclusive Financial Sectors instó a los bancos centrales y a los países para adicionar el objetivo de la inclusión financiera a los dos objetivos tradicionales del sistema financiero: estabilidad y regulación prudencial para la seguridad de los fondos de los depositantes (Kumar, Narain \& Rubbani, 2015).

El Reino Unido ha creado un fondo de inclusión financiera para promoverla y ha asignado la responsabilidad a los bancos y cooperativas de crédito en la eliminación de la exclusión financiera (Leeladhar, 2006). Ahora bien, en Estados Unidos también se ha trabajado sobre la inclusión financiera, pues mediante la Ley de Reinversión Comunitaria se ha tomado acción al extender la prestación de servicios bancarios y de crédito a todos los sectores de la sociedad (Estados Unidos, 1977).

La inclusión financiera ha sido definida de varias maneras por diferentes autores; hay definiciones muy sencillas, como la de Musa Abdullahi Bayero (2015): es la incapacidad de acceder a servicios financieros apropiados. La de Franklin Allen, Asli Demirgüç-Kunt, Leora Klapper y María Soledad Martínez-Peria (2016): es el uso de los servicios financieros formales. Para Lindsey Appleyard (2011), la inclusión financiera es cuando los empresarios y las empresas pueden abrir una cuenta bancaria y tener acceso a facilidades de crédito asequibles como un sobregiro o tarjeta de crédito.

La definición siguiente — del Committee on Financial Inclusion (CFI) - es algo más compleja: proceso de garantizar el acceso a los servicios financieros y de crédito, de manera oportuna y adecuada, cuando lo necesiten los sectores más débiles de una economía y los grupos de bajos ingresos; a un costo asequible (CFI, 2008). Para Usha Thorat (2006), la inclusión financiera implica la prestación de servicios financieros 
asequibles, servicios como el acceso a los pagos y las instalaciones de remesas, ahorros, préstamos y servicios de seguros por parte de los sistemas financieros formales a los que tienden a ser excluidos.

Según Nidia García, Andrea Grifoni, Juan Carlos López y Diana Margarita Mejía (2013, p. 22), la inclusión financiera es definida por la INFE [International Network on Financial Education, Red Internacional de Educación Financiera] como:

El proceso de promoción de un acceso asequible, oportuno y adecuado a una amplia gama de productos y servicios financieros regulados y la ampliación de su uso por todos los segmentos de la sociedad, a través de la aplicación de enfoques innovadores o existentes hechos a la medida, incluyendo actividades de sensibilización y de educación financiera, con el fin de promover el bienestar económico y la inclusión económica y social.

Teniendo en cuenta lo anterior, se puede determinar que más que una definición como tal se debe hablar del concepto de inclusión financiera, concepto multidimensional que incluye como mínimo un mejor acceso, mejores productos y servicios, y un mejor uso de ellos; para Sharit K. Bhowmik y Debdulal Saha (2013), los puntos más importantes sobre el concepto de inclusión financiera son:

- Es difícil de definir y de medir.

- Las personas de todos los estratos de la sociedad deben tener acceso a los servicios financieros formales.

- No debe haber barreras de acceso al crédito formal.

- Cantidad oportuna y adecuada de crédito debe ser proporcionada.

- Las personas de ingresos bajos y débiles necesitan ser dirigidas.

- Los servicios financieros tienen que ser baratos y asequibles.

Ahora bien, la Declaración Maya [ primera serie mundial de compromisos medibles para aumentar el acceso a los servicios financieros formales para 2.500 millones de personas no bancarizadas del mundo; declaración elaborada y aprobada por los miembros de la Alliance for Financial Inclusion, AFI, en la Riviera Maya, México en 2011] (AFI, 2011) se basa en un concepto de complejidad mediante el establecimiento de una orientación política general de cuatro áreas interconectadas para evaluar el avance de la inclusión financiera (AFI, 2013):

- Creación de un entorno propicio para aprovechar la nueva tecnología.

- Implementación de un marco regulador equilibrado.

- Protección de la armonización del consumidor y la educación.

- Acopio y utilización de datos de inclusión financiera.

\section{Medición de la inclusión financiera}

Para Peter Morgan y Victor Pontines (2015), las medidas de inclusión financiera incluyen muchas ideas, como el número de sucursales bancarias, cuentas bancarias, cajeros automáticos por cada mil personas; empresas con depósitos o línea de crédito, porcentaje de las pyme en relación con el total de depósitos o líneas de crédito; adultos con depósito en instituciones financieras formales y adultos prestatarios. Cecilia Vera y Daniel Titelman (2013), en publicación para la Comisión Económica para América Latina -CEPAL-, sostienen que algunos de los indicadores usados para medir la inclusión financiera, son aquellos que miden el acceso a los servicios financieros: proporción de adultos en la economía que declaran tener una cuenta en una institución financiera formal, porcentaje de individuos mayores de 15 años de edad que utiliza tarjetas de crédito o de débito, porcentaje de empresas que cuentan con un préstamo o línea de crédito diferenciadas por el tamaño.

El Grupo de los Veinte -G20 - ha venido reconociendo el papel clave de la inclusión financiera en la lucha contra la pobreza y en la búsqueda de un desarrollo inclusivo, tanto es así que ha propuesto se mida en tres dimensiones: (i) el acceso a los servicios financieros; (ii) el uso de los servicios financieros; y (iii) la calidad 
de los productos y la prestación de servicios. Tanto los datos de la oferta como los de la demanda se incluyen para formar una visión global. Global Partnership for Financial Inclusion, GPFI (2016) propone un grupo de 24 indicadores ( 8 de ellos propuestos en 2016): adultos con una cuenta, número de cuentas, adultos con crédito en una institución regulada, adultos con seguro, transacciones sin dinero en efectivo, adultos que usan pagos digitales, pagos que usan teléfonos móviles (desde una cuenta), pagos por internet, pago con una tarjeta bancaria, pago desde una cuenta, alta frecuencia de uso de cuenta, propensión al ahorro, empresas en bancos formales, empresas con préstamo pendiente o línea de crédito en instituciones reguladas, pagos digitales desde o hacia empresas, puntos de servicio, tarjeta débito, puntos de servicio de empresas, interoperatividad de los puntos de servicio, conocimiento financiero, comportamiento financiero, información de requisitos, resolución de reclamos y barreras crediticias.

El objetivo del Brookings Financial and Digital Inclusion Project -FDIP- es proporcionar a los legisladores, al sector privado, a los representantes de organizaciones no gubernamentales y al público en general la información que puede ayudar a mejorar la inclusión financiera en sus respectivos países; para dar cumplimiento a este objetivo ha diseñado 33 indicadores que abarcan cuatro "dimensiones" que representan lo que ellos consideran como áreas claves asociadas con el acceso y uso de los servicios financieros: compromiso de los países, la capacidad móvil, entorno normativo y la adopción (Villasenor, West \& Lewis, 2016). Con respecto a la primera dimensión, compromiso de los países, se examina el papel del gobierno (en particular del regulador nacional u otra autoridad del sector público en cuanto a inclusión financiera) como motor que propicia las condiciones para la inclusión financiera; teniendo en cuenta la capacidad de cada gobierno para recopilar los datos a escala nacional, coordinar con los miembros del sector privado, y permitir el desarrollo de un marco regulatorio; reconociendo que el sector público desempeña un importante papel en la promoción de la inclusión financiera. En la capacidad móvil, la segunda dimensión, están indicadores que miden la infraestructura móvil y su adopción, así como indicadores específicos para dinero móvil. Entre los indicadores específicos utilizados para evaluar el entorno regulatorio están los agentes bancarios, los servicios móviles financieros no bancarios (con un enfoque en el rol de la red de operadores de telefonía móvil), los reglamentos de dinero electrónico, la plataforma de dinero móvil proporcionada a los clientes y la capacidad de las agencias para retiro en efectivo. La última dimensión de la evaluación se centra en la adopción de servicios financieros tradicionales y digitales (Villasenor, West \& Lewis, 2015).

Ahora bien, el índice propuesto por AFI para medir la inclusión financiera en cada país maneja cuatro dimensiones: acceso, uso, calidad y bienestar (AFI, 2016). En estudios realizados por The Economist Intelligence Unit, analizan el entorno para la inclusión financiera y evalúan el ambiente normativo con base en 12 indicadores (The Economist Intelligence Unit, 2014). Ahora bien, el Grupo del Banco Mundial (World Bank Group, 2015) presenta una metodología con 9 indicadores y 41 subindicadores para cada país.

En lo que respecta a estudios para cada país, la investigación realizada por Satya R. Chakravarty y Rupayan $\mathrm{Pal}$ (2013) examina los efectos de las principales políticas de la banca en la inclusión financiera en la India entre 1972 y 2009, utilizando técnicas de econometría de datos de panel. En su artículo, Pallavi Chavan (2007) examina el grado de acceso al crédito formal de los hogares rurales en uno de los sectores más atrasados de la población en la India, con datos extraídos de encuestas oficiales y manejados de manera tradicional respecto a porcentajes y tendencias. También para la India, Rajani Gupte, Bhama Venkataramani y Deepa Gupta (2012, p. 133) se centran en un cálculo de indicadores tradicionales a partir de los datos del Banco Mundial para este país.

De otra forma, Gonzales D'Alcantara y Axel Gautier (2013) estudian el papel que los operadores postales pueden jugar en la inclusión financiera; se parte del hecho de que en los países en desarrollo las empresas postales normalmente tienen una red de oficinas con una cobertura que se extiende a todo el territorio, mientras que el sector bancario está completamente ausente en las zonas rurales; el modelo propuesto se basa en la cuantificación de los costes y los beneficios de la asociación. En otra investigación, Asli Demirgüç-Kunt y Leora Klapper (2013) estudian cómo los adultos usan los sistemas financieros formales e informales para 
gestionar su día a día financiero y planificar el futuro; los datos muestran que el 50 por ciento de los adultos de todo el mundo están "bancarizados", es decir, tienen una cuenta en una institución financiera formal.

Adicionalmente, la investigación hecha por Eduardo Diniz, Rene Birochi y Marlei Pozzebon (2012) presenta un estudio de caso de la inclusión financiera en Autazes, un condado de Brasil en la región amazónica no atendido por la banca hasta 2002, cuando un corresponsal inició sus operaciones en ese país; los datos fueron categorizados mediante el software NVivo para su condensación.

China también ha sido objeto de estudio. Zuzana Fungáčová y Laurent Weill (2015) realizaron una investigación sobre la inclusión financiera utilizando los indicadores del Banco Mundial y encontraron un mayor uso de las cuentas de ahorro formal y un uso menos frecuente del crédito.

Para Kenia, Susan Johnson y Steven Arnold (2012) analizaron la situación de factores socioeconómicos, demográficos y geográficos asociados con el uso de servicios financieros formales, semiformales e informales entre 2006 y 2009, usando la técnica de regresión de Probit.

\section{En Colombia}

Como resultado de las recomendaciones de política expuestas en el documento Conpes 3424 de 2006 (Colombia, 2006), se expide el Decreto 3078 de 2006 (Colombia, 2006), que creó el Programa de Inversión Banca de las Oportunidades, y explicita sus actividades y los recursos asignados.

La Banca de las Oportunidades - BdO- y la Superintendencia Financiera de Colombia -SFC- han tenido la responsabilidad de generar y publicar anualmente los informes de inclusión financiera. En el primero de ellos, para 2011, la BdO y la SFC (2012, p. 9) consignan:

En los últimos años, la inclusión financiera ha adquirido gran importancia a nivel mundial, tanto en las agendas de gobierno, especialmente de países en vía de desarrollo, como a través de iniciativas multilaterales, reconociéndose de este modo la importancia de lograr una mayor inclusión financiera como herramienta de lucha contra la pobreza, así como para una mejor distribución de ingresos y el fomento del desarrollo económico.

En esta primera versión, la BdO y la SFC recogen información desde 2008 y hasta 2011, y definen la inclusión financiera como "el acceso y utilización de servicios financieros formales por parte de las actividades económicas y de la población” (2012, p. 14). Adicionalmente, acogen la propuesta de la AFI respecto a las dimensiones de la inclusión financiera. Presentan como método de medición dos indicadores manejados de manera global:

- De acceso: Puntos de contacto y cobertura geográfica de los puntos de acceso.

- De uso: Indicador de bancarización, productos financieros pasivos, productos financieros activos y canales transaccionales.

En el segundo informe, la BdO y la SFC (2013) continúan manejando los mismos indicadores y presentan un aparte sobre protección al consumidor financiero.

Para 2013, continúan con los mismos indicadores y adicionan dos tipos de análisis: por puntos de contacto y por tipo de institución financiera. Las 236 instituciones financieras analizadas globalmente son bancos, compañías de financiamiento, cooperativas financieras, cooperativas de ahorro y crédito y organizaciones no gubernamentales -ONG- microcrediticias (BdO \& SFC, 2014, p. 31).

Se parte del hecho de que en el sistema financiero colombiano hay dos organismos oficiales que supervisan los agentes que desarrollan actividades financieras:

1. La Superintendencia Financiera de Colombia, cuyas principales funciones son: Ejercer la inspección, vigilancia y control sobre las personas que realicen actividades financieras, bursátiles, aseguradoras y cualquier otra relacionada con el manejo, aprovechamiento o inversión de recursos 
captados del público con el fin de preservar su estabilidad, seguridad y confianza, así como promover, organizar y desarrollar el mercado de valores colombiano y la protección de los inversionistas, ahorradores y asegurados. Entre las entidades que vigila esta entidad están los bancos, compañías de financiamiento, cooperativas financieras y compañías de seguros (SFC, 2016).

2. La Superintendencia de Economía Solidaria -SES-, la cual: "vigila a las cooperativas de ahorro y crédito y ejerce el control, inspección y vigilancia protegiendo los intereses de los asociados de las organizaciones de economía solidaria, de los terceros y de la comunidad en general" (SES, 2016).

La diferencia fundamental entre estas dos entidades es el tipo de instituciones financieras que vigilan. La SFC vigila a:

- Bancos: institución financiera que tiene como función principal captar recursos del público mediante depósitos a la vista y depósitos a término, para nuevamente colocarlos en forma de préstamos, descuentos, anticipos u otras operaciones activas de crédito.

- Compañías de financiamiento: instituciones cuya función principal consiste en captar recursos a término, con el objeto fundamental de realizar operaciones activas de crédito para facilitar la comercialización de bienes y servicios, y ejecutar operaciones de arrendamiento financiero o leasing.

- Cooperativas financieras: organismos cooperativos especializados cuya función principal consiste en adelantar actividades financieras para ofrecer sus servicios a terceros no asociados.

Mientras que la SES vigila a las cooperativas de ahorro y crédito: organismos cooperativos especializados cuya función principal consiste en adelantar actividad financiera exclusivamente con sus asociados.

En 2014, estudiaron 229 entidades de los mismos tipos que para el año inmediatamente anterior (BdO \& SFC, 2015); adicionalmente, presentan una síntesis de los principales resultados de Colombia en materia de inclusión financiera a la luz de algunos reportes internacionales como el Microscopio 2014 y la encuesta de demanda del Global Findex 2014; y algunos indicadores de protección al consumidor, a partir del análisis de la información sobre las quejas y reclamos que gestiona la Superintendencia Financiera de Colombia.

De otra manera es importante resaltar que según el Consejo Privado de Competitividad, Compite: "El aumento en el acceso a productos financieros es el resultado de la consolidación de la política de inclusión financiera. El país ha avanzado en materia de inclusión financiera y las entidades del sector público han implementado medidas que buscan fortalecer su desempeño" (2016, p. 146).

En la investigación realizada por el Center for Financial Inclusion (CFI, 2015), mediante una metodología de mesa redonda con expertos, sobre envejecimiento e inclusión financiera en Colombia en noviembre de 2014, ellos "vieron oportunidades para vincular algunos servicios financieros de apoyo específicos transferencias de dinero, crédito de empresa y consumo, productos de ahorro a largo plazo y seguro- con las pensiones, para ampliar la gama de herramientas financieras a las que las personas mayores pueden tener acceso"; adicionalmente, detectaron una falta de infraestructura de servicios financieros, en especial en áreas rurales, como una barrera específica a la inclusión financiera de este grupo.

Por todo lo anterior se ha mostrado que, por una parte, la inclusión financiera es un tema de gran importancia tanto en el ámbito nacional como en el internacional; y por otra parte, que diferentes instituciones e investigadores se han preocupado de medirla mediante diferentes tipos de indicadores; pero siempre indicadores tradicionales que se manejan de manera global y no por dependencia o entidad. De esta manera, esta investigación analiza la situación y evolución de la inclusión financiera en Colombia desde un punto de vista diferente: desde la eficiencia relativa; manejando las entidades financieras como objeto específico, no global, de análisis. Por esto, se considera que esta investigación es pionera. Se generan los indicadores de eficiencia relativa de inclusión financiera de 2013 y 2014, y se comparan. La metodología que se utiliza es Data Envelopment Analysis (DEA), metodología de programación lineal avanzada que mediante 
un doble proceso de optimización proporciona un único índice de eficiencia relativa para cada una de las unidades estudiadas, con la fortaleza de analizar las variables en un escenario de utilización de multirrecursos y de obtención de multisalidas. Se da respuesta a los siguientes interrogantes: ¿Qué tipo de entidades es eficiente y en qué número, para cada año? ¿Qué resultados se obtienen cuando se compara el comportamiento, en términos de eficiencia relativa, de 2013 y 2014 ?

\section{Metodología}

\section{Data Envelopment Analysis, DEA, Análisis Envolvente de Datos}

Es un método no paramétrico usado para evaluar la eficiencia relativa de una unidad que toma decisiones (Decision Making Unit, DMU), a partir de la generación de una frontera eficiente mediante la ubicación de los índices relativos individuales, cuando no se conocen las relaciones funcionales entre los recursos y los productos obtenidos en un proceso de transformación (Chediak-Pinzón, 2008).

La medida relativa se deriva de la medida universal de eficiencia (Cooper, Seiford \& Tone, 2000, p. 3):

salida/entrada

De otra parte, DEA es una metodología que se utiliza para evaluar la eficiencia relativa de un conjunto de $n$ DMU, mediante el planteamiento de problemas de programación lineal para cada unidad de acuerdo con los datos observados de entradas (recursos) y salidas (productos) (Wei \& Yan, 2010, p. 612). Cuando se quiere establecer la eficiencia relativa, es importante aclarar que la eficiencia o ineficiencia de una DMU específica está dada tanto por su propia ejecución como por la ejecución de las otras, pues aun cuando el punto es que las DMU seleccionadas sean homogéneas, cada una tiene características que la hacen única (Rodríguez-Lozano \& Tarazona-Morales, 2015). DEA ha experimentado un rápido desarrollo, hasta convertirse progresivamente en un conjunto de conceptos y metodologías, los cuales se han concretado en un abanico de modelos (Rodríguez-Lozano, 2011). Según Ki-Hoon Lee y Reza Farzipoor Saen (2012), esta metodología no requiere asumir previamente una función de producción para ser aplicada. Una de las fortalezas de esta metodología es que establece el valor numérico de la ineficiencia (Cook, Kress \& Seiford, 1993).

Los modelos tradicionales de DEA proporcionan una clasificación categórica de la DMU en eficiente o ineficiente (Iyer \& Banerjee, 2016).

Hay dos modelos básicos DEA: Constant Return to Scale, CRS y Variable Return to Scale, VRS (Quintanilha da Silveira, Correia Baptista Soares de Mello \& Angulo-Meza, 2012). En el modelo CRS, cualquier variación en las entradas produce una variación en la misma proporción en las salidas, lo que genera retornos constantes de escala; por el contrario, el modelo VRS no asume proporcionalidad en las variaciones de entradas y salidas, lo que genera retornos variables de escala (Cervera-Muñoz, Oviedo-García \& Pineda-Acero, 2013). En el modelo VRS, los retornos a escala pueden ser adicionalmente incrementales y decrementales (Lau, 2013).

\section{Modelo CRS}

Considere un conjunto compuesto por $n$ DMU denotadas como $\operatorname{DMU}_{\mathrm{j}}(\mathrm{j}=1, \ldots, \mathrm{n})$ las cuales utilizan recursos $\mathrm{x}_{\mathrm{ij}}(\mathrm{i}=1, \ldots, \mathrm{m})$ y generan $s$ salidas $\mathrm{y}_{\mathrm{rj}}(\mathrm{r}=1, \ldots, \mathrm{s})$, y partiendo de que los multiplicadores $v_{i}, u_{r}$ asociados con $i$ entradas y $r$ salidas respectivamente son conocidos. Entonces, específicamente, si la DMU está bajo estudio, este modelo está dando la solución del problema de programación fraccional para la medida de eficiencia de esa $\mathrm{DMU}_{0}$ así (Rodríguez-Lozano, 2011, p. 74): 


$$
\sum_{r} u_{r} y_{r j}-\sum_{i} v_{i} x_{i j} \leqslant 0
$$

Sujeto a:

$\sum_{,} u_{y_{j}}-\sum_{i} v_{x, j} \leqslant 0$, para todo $j$

$u_{r}, v_{i} \geqslant \varepsilon$, para todo $r, i$

Donde $\varepsilon$ es un valor no- arquimidian designado estrictamente positivo.

Aplicando la teoría de programación fraccionaria expresada en Abraham Charnes y William W. Cooper (1962) y realizando los siguientes cambios de variables:

$$
\mu_{r}=t u_{r} \mathrm{y} v_{i}=t v_{i}
$$

donde:

$$
t=\left(\sum_{i} v_{i} x_{i o}\right)^{-1}
$$

El problema inicial puede ser transformado en el siguiente modelo de programación lineal:

$$
e_{0}=\max \sum_{r} \mu_{r} y_{r o}
$$

Sujeto a:

$$
\begin{aligned}
& \sum_{i} v_{i} x_{i o}=1 \\
& \sum_{r} \mu_{r} y_{r j}-\sum_{i} v_{i} x_{i j} \leqslant 0, \quad \forall j
\end{aligned}
$$

$\mu_{r}, v_{i} \geqslant \varepsilon$ para todo $r, i$

\section{Modelo VRS}

Retomando todos los parámetros anteriores, para este modelo se tiene como planteamiento matemático (Rodríguez-Lozano, 2011, p. 76):

$$
e_{o}^{*}=\max \quad\left[\sum_{r} u_{r} y_{r o}-u_{o}\right] / \sum_{i} v_{i} x_{i o}
$$

Sujeto a:

$$
\begin{aligned}
& \sum_{\mathrm{r}} u_{\mathrm{r}} y_{r j}-u_{o}-\sum_{i} v_{i} x_{i j} \leqslant 0, \quad j=1, \ldots, n \\
& u_{r} \geqslant \varepsilon, \quad v_{i} \geqslant \varepsilon, \quad \forall i, r
\end{aligned}
$$

$u_{O}$ no restringida en signo

Con su equivalente en programación lineal: 


$$
e_{o}^{*}=\max \sum_{r} \mu_{r} y_{r o}-\mu_{o}
$$

Sujeto a:

$$
\begin{aligned}
& \sum_{\mathrm{i}} v_{\mathrm{i}} x_{i o}=1 \\
& \sum_{r} \mu_{r} y_{r j}-\mu_{o}-\sum_{i} v_{i} x_{i j} \leqslant 0, \quad j=1, \ldots, n \\
& \mu_{r} \geqslant \varepsilon, \quad v_{i} \geqslant \varepsilon, \quad \forall i, r
\end{aligned}
$$

$\mu_{o}$ irrestricta

Tanto el modelo CRS como el modelo VRS se pueden trabajar con orientación a las entradas o a las salidas. En la orientación a las entradas o recursos se busca la máxima disminución en el nivel de entradas mientras el nivel de producción permanece estable. La orientación a las salidas busca la máxima respuesta posible de las salidas manteniendo el nivel de las entradas. En este sentido, una unidad no puede ser caracterizada como eficiente si es posible incrementar cualquier salida sin incrementar ninguna entrada y sin disminuir ninguna otra salida (Coll \& Blasco, 2006). Desde esta misma óptica, Marcela C. González-Araya y Nelson G. Valdés (2009), Gang Lee, Ming-Miin Yu y Lung-Chuang Wang (2012) coinciden en que el primer desafío, al aplicar modelos DEA, es seleccionar las variables que describan con mejor precisión el proceso de transformación que desarrollan las DMU evaluadas.

Para la aplicación de DEA, es importante tener en cuenta que las DMU elegidas cumplan las siguientes condiciones: homogeneidad, para que se puedan comparar; heterogeneidad: para que se pueda extraer información de la comparación; y que tengan alguna capacidad para gestionar los recursos de los que disponen (Pino-Mejías, Solís-Cabrera, Delgado-Fernández \& Barea-Barrera, 2010).

\section{Revisión de la literatura actual}

Aunque hay una gran variedad de literatura referente a la aplicación de la metodología DEA en el sector real y en el sector financiero, no hay investigaciones referentes a inclusión financiera y al estudio de la eficiencia relativa de entidades financieras en este contexto. Sin embargo, se presentan algunas investigaciones actuales con DEA, adelantadas en el sector financiero.

En este sector fianciero, el sistema bancario y los bancos son los temas más recurrentes en el estudio de la eficiencia relativa mediante método no paramétrico. Ejemplo de esto es la investigación de Zuzana Svitálková (2014) que compara la eficiencia del sistema bancario de República Checa, Eslovaquia, Austria, Polonia, Hungría y Eslovenia; utilizando los modelos VRS y CRS, este estudio abarca solo bancos comerciales. Las variables de entrada estudiadas fueron costos de personal, depósitos y activos fijos; y las de salida ingresos netos por intereses, créditos totales, provisión de créditos incobrables. Como resultado del trabajo, se estableció que los sectores bancarios austriaco y checo tienen el mejor rendimiento, y los sistemas más ineficientes fueron los de Eslovenia y Eslovaquia.

Jolly Puri y Shiv Prasad Yadav (2014) estudian el sector bancario de la India. Ellos proponen un modelo DEA difuso y con salidas no deseadas pues los datos de las variables de entrada y de salida no siempre están disponibles de manera exacta, es decir, son difusos. Consideran 12 DMU, dos variables de entrada difusas (número de empleados y depósitos totales) y dos variables de salida difusas, siendo una de estas una salida difusa no deseable (créditos no morosos y créditos morosos). Los resultados obtenidos muestran, al decir de 
estos investigadores, que el impacto de las variables de salida no deseables permite analizar de manera eficiente la presencia de incertidumbre en los datos, por medio de los resultados de eficiencia.

Para este país también se realizó un estudio mediante el modelo DEA basado en las holguras (Slacks-based measure, SBM), que es una medida para estimar qué tan bien se utiliza el conjunto de entradas o de salidas. Jolly Puri y Shiv Prasad Yadav (2013, p. 1439) utilizan este modelo pues parten del hecho de que en los problemas del mundo real, los datos pueden ser imprecisos o difusos. El modelo es aplicado al Banco del Estado de Patiala en el estado de Punjab de la India con los distritos como DMU.

En el caso específico de estudio de los bancos que apoyan la construcción en China, el trabajo de Zhongbao Zhou, Liang Sun, Wenyu Yang, Wenbin Liu y Chaoqun Ma (2013) utiliza el modelo de juego de negociación del matemático estadounidense John Forbes Nash, premio Nobel de Economía en 1994, para la descomposición de la eficiencia en el modelo centralizado de dos etapas DEA, en el cual todas las salidas de la primera etapa son las únicas entradas a la segunda. Se aplicó este modelo para evaluar el rendimiento de 10 sucursales de los bancos ya mencionados.

También para los bancos islámicos se realizó una investigación utilizando DEA (Johnes, Izzeldin \& Pappas, 2014); adicionalmente, para comparar los resultados con bancos tradicionales, se utilizó el metaanálisis de frontera (meta-frontier analysis, MFA); puesto que este último permite la descomposición de la eficiencia en dos componentes: eficiencia neta (la eficiencia de los bancos se mide en relación con su propio tipo de frontera) y la eficiencia global (la eficiencia que se refiere a modus operandi). Se encontró que los bancos islámicos suelen estar por debajo de los convencionales en términos de eficiencia global y significativamente por encima en la eficiencia neta. La baja eficiencia de los bancos islámicos podría atribuirse a la normalización de los productos, mientras la alta eficiencia neta obedece a la gestión en los bancos.

En Canadá, Abaghan Ghahraman y Diego Prior (2016) realizaron un estudio para medir la eficiencia de 79 sucursales bancarias a partir de un modelo DEA VRS con orientación a las entradas que ellos denominaron de red, al agrupar previamente los DMU bajo estudio.

Para Brasil, Peter Wanke y Carlos Barros (2014) realizaron un estudio en el cual efectúan la medición de la eficiencia en 40 bancos en 2012, con un modelo de dos etapas, para poder modificar las variables de entrada. Para optimizar ambas etapas simultáneamente, los autores utilizan el modelo centralizado de eficiencia. El resultado del estudio muestra que los bancos brasileños son heterogéneos: algunos se centran en la eficiencia de costos (primera etapa) y otros en la eficiencia productiva (segunda etapa).

Teniendo como objeto de estudio la banca colombiana, en la investigación realizada por Miguel Sarmiento, Andrés Cepeda, Hernando Mutis y Juan Pérez (2014), los autores evalúan la eficiencia relativa de 23 bancos comerciales entre 2000 y 2009, utilizando los modelos CRS y VRS orientados a entradas y a salidas. Plantean como entradas: depósitos y costos totales. Como salidas: inversiones y cartera en créditos, para el análisis de la eficiencia técnica. Para el análisis de eficiencia de costos, plantean tres entradas: capital laboral, capital financiero y capital físico; y dos salidas: inversiones y créditos ofrecidos. Como resultado, el estudio establece que hay incremento de la eficiencia bancaria y al mismo tiempo una alta heterogeneidad de las medidas de eficiencia entre las DMU analizadas.

Coincidiendo con el objeto de estudio de la investigación anteriormente mencionada, la investigación de Javier E. Pirateque, José H. Piñeros y Linda Mondragón (2013, p. 9) analiza la eficiencia de la banca colombiana entre 2000 y 2012; y encuentra que las entidades nacionales son más eficientes que las extranjeras. Se estudiaron 18 establecimientos bancarios que fueron comparados mediante el modelo VRS orientado a salidas.

Mehdi Toloo (2015) propone un nuevo modelo y sugiere que una de sus aplicaciones podría ser la medición de la industria bancaria, tomando como DMU las sucursales. La propuesta de modelo se basa en programación lineal entera mixta que es capaz de determinar la unidad más eficiente bajo una condición común. 


\section{Diseño metodológico}

Para esta investigación, las fuentes de información son la Superintendencia Financiera de Colombia y Banca de las Oportunidades. Para 2013, se analizaron 52 DMU: 24 bancos, 23 compañías de financiamiento y 5 cooperativas financieras; para 2014, también se analizaron 52 DMU: 23 bancos, 23 compañías de financiamiento y 6 cooperativas financieras. Los datos corresponden al 100\% de la información extractada de las fuentes ya mencionadas.

Dado que no se encontró evidencia de la existencia de retornos a escala constantes, se optó por utilizar el modelo DEA VRS (Banker, Charnes \& Cooper, 1984).

Para este modelo se establecieron cuatro entradas y tres salidas.

Entradas

Número de oficinas de atención al público

Número de cajeros

Número de datafonos

Número de corresponsales bancarios

Salidas

Número de operaciones no monetarias

Número de operaciones monetarias

Monto de las operaciones monetarias

\section{Resultados}

\section{Año 2013}

Los resultados del número de DMU eficientes se muestran en la tabla 1 y en el gráfico 1 .

TABLA 1

DMU eficientes

\begin{tabular}{|l|c|}
\hline \multicolumn{2}{|c|}{ DMU eficientes año 2013 } \\
\hline Bancos & 12 \\
\hline Compañías de financiamiento & 6 \\
\hline Cooperativas financieras & 0 \\
\hline
\end{tabular}

Fuente: elaboración propia 


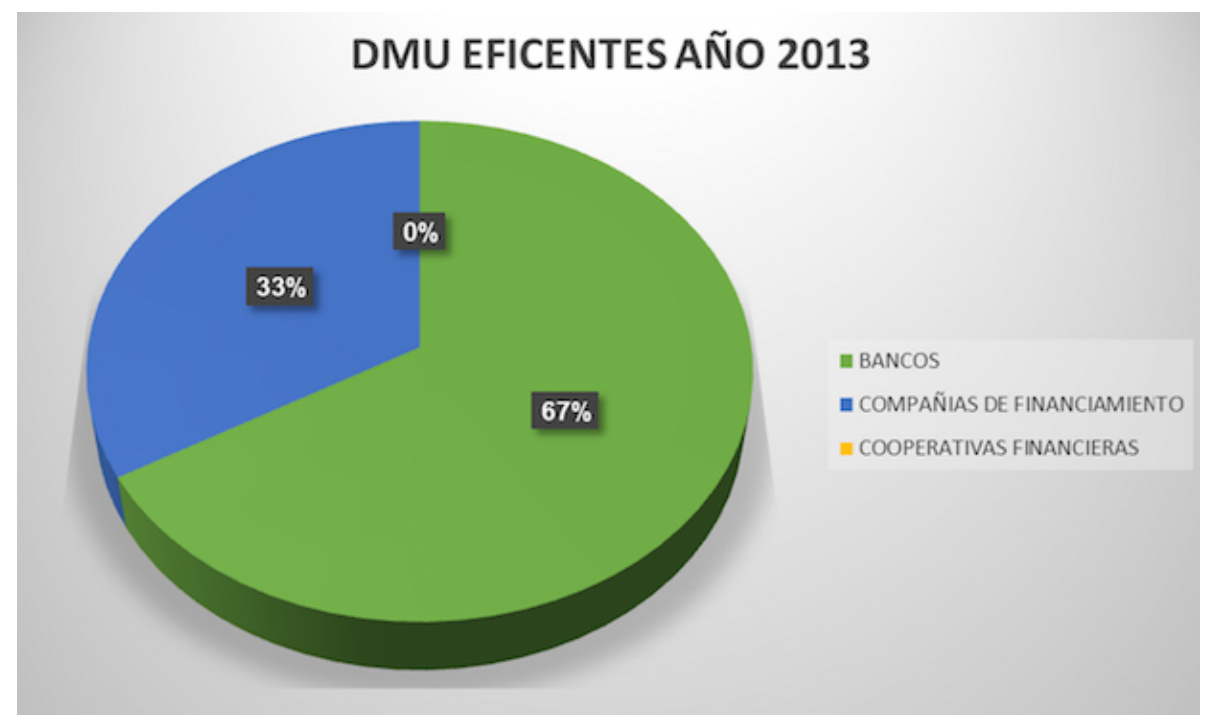

FIGURA 1

DMU eficientes

Fuente: elaboración propia

\section{Año 2014}

Los resultados del número de DMU eficientes se muestran en la tabla 2 y en el gráfico 2.

TABLA 2

DMU eficientes

\begin{tabular}{|l|c|}
\hline \multicolumn{2}{|c|}{ DMU eficientes año 2014 } \\
\hline Bancos & 11 \\
\hline Compañías de financiamiento & 2 \\
\hline Cooperativas financieras & 0 \\
\hline
\end{tabular}

Fuente: elaboración propia 


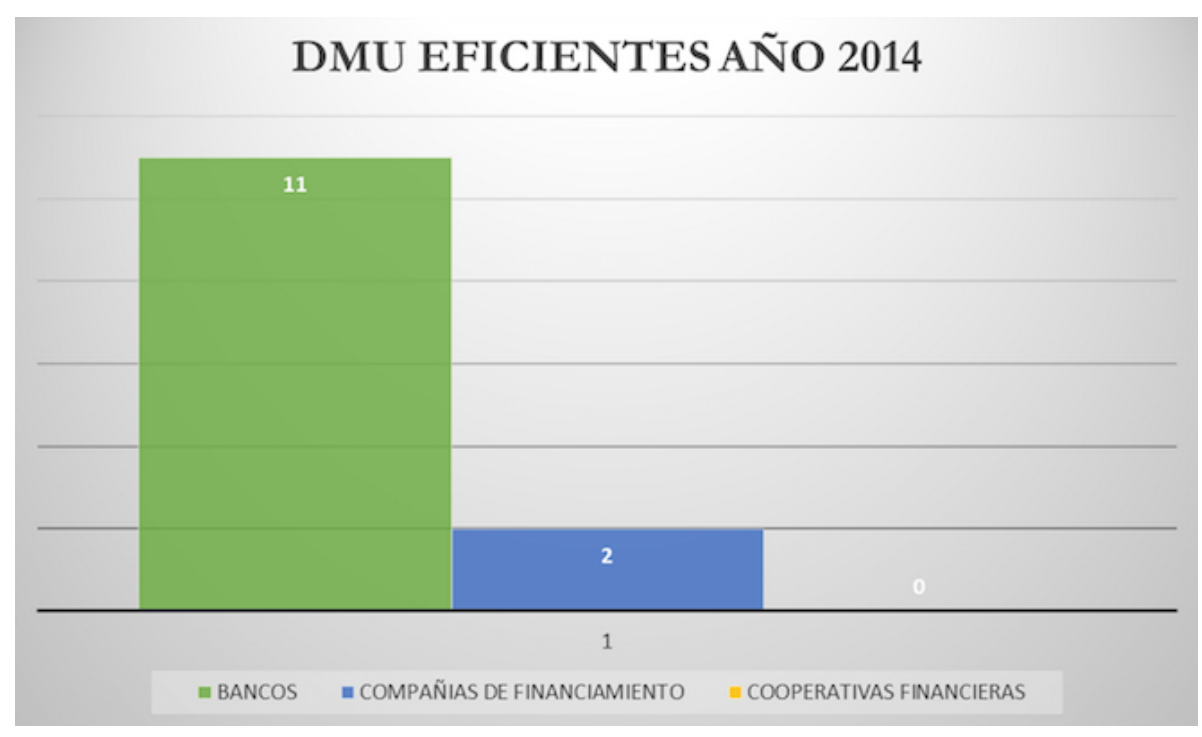

FIGURA 2

DMU eficientes

Fuente: elaboración propia

\section{Comparativo}

En la tabla 3 y gráfico 3 se muestra el número de unidades para cada grupo de valores de eficiencia relativa.

TABLA 3

Comparativo eficiencia DMU

\begin{tabular}{|l|r|r|r|r|}
\hline \multicolumn{5}{|c|}{ Modelo VRS } \\
\hline EF (\%) & 20 & 13 & 20 & 14 \\
\hline 100 & 18 & $35 \%$ & 13 & $25 \%$ \\
\hline $99,9-80$ & 4 & $8 \%$ & 3 & $6 \%$ \\
\hline $79,9-60$ & 0 & $0 \%$ & 1 & $2 \%$ \\
\hline $59,9-40$ & 5 & $9 \%$ & 5 & $10 \%$ \\
\hline $39,9-0,1$ & 25 & $48 \%$ & 10 & $19 \%$ \\
\hline 0 & 0 & $0 \%$ & 20 & $38 \%$ \\
\hline
\end{tabular}

Fuente: elaboración propia 


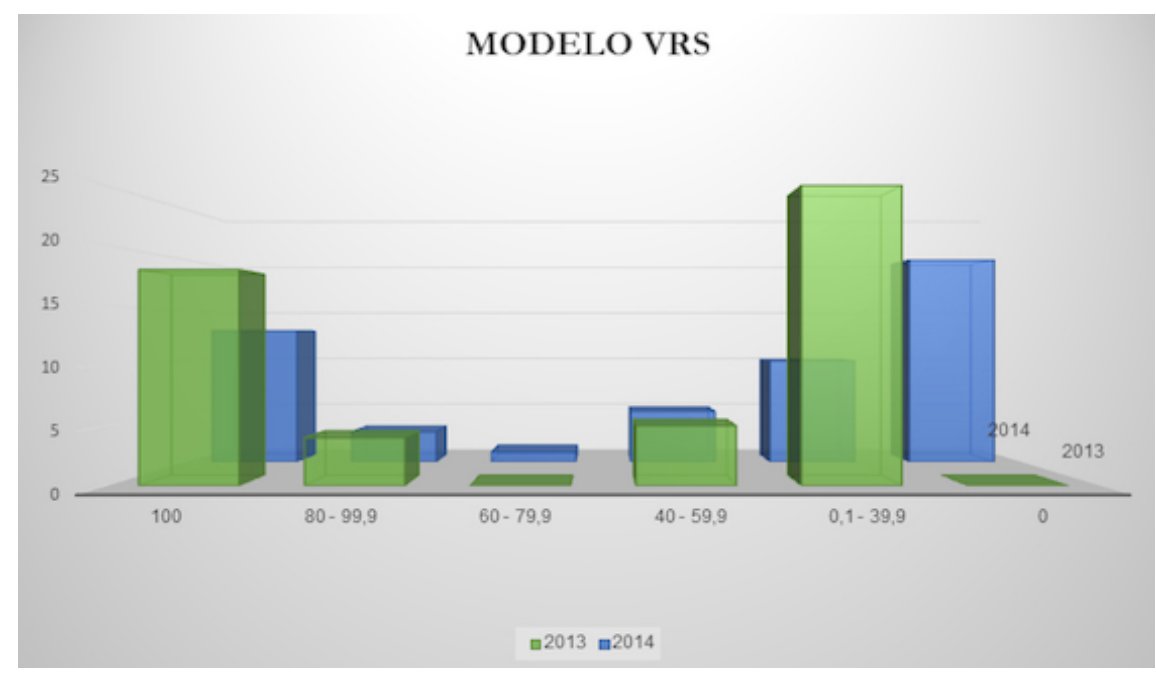

FIGURA 3

Comparativo eficiencia DMU

Fuente: elaboración propia

Se observa que hay un desmejoramiento en la cantidad de unidades eficientes, pues pasan del $35 \%$ al $25 \%$. Adicionalmente, para los índices menores de 40\%, el porcentaje de unidades pasa del $48 \%$ al $57 \%$, lo que aumenta los malos resultados; de tal manera que globalmente se evidencia un desmejoramiento en los resultados de 2013 a 2014.

Solo 10 DMU son eficientes en ambos años, de estas 10, el 80\% son bancos; entre las DMU eficientes, nunca hubo ninguna cooperativa.

Para 2013: (gráfico 4)

- De los 24 bancos, 12 (50\%) son eficientes.

- De las 23 compañías de financiamiento, $6(26 \%)$ son eficientes.

- De las 5 cooperativas financieras, ninguna $(0 \%)$ es eficiente.

Para el año 2014: (gráfico 4)

- De los 23 bancos, 11 (48\%) son eficientes.

- De las 23 compañías de financiamiento, 2 (9\%) son eficientes.

- De las 6 cooperativas financieras, ninguna $(0 \%)$ es eficiente. 


\section{DMU EFICIENTES (\%)}

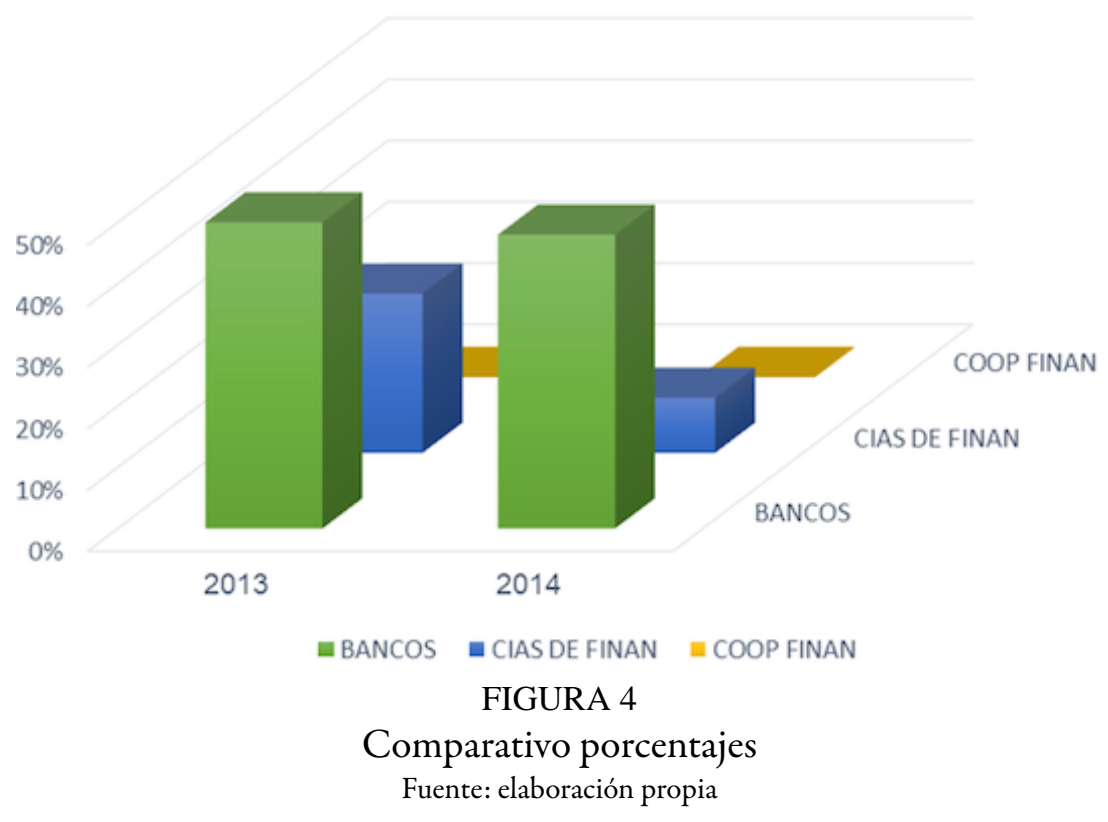

\section{Conclusiones}

Globalmente, se evidencia un desmejoramiento en el comportamiento de la eficiencia relativa de las instituciones evaluadas en el escenario de la inclusión financiera: de 2013 a 2014, disminuyen las unidades eficientes y aumentan las unidades con bajos índices.

1. El total de entidades eficientes para 2014 (13) es menor que para 2013 (18).

2. Los bancos son las unidades que más contribuyen a los altos índices:

a. Para índices mayores de 80, hay 16 bancos (67\%) año 2013

b. Para índices mayores de 80 , hay 13 bancos (57\%) año 2014

3. El banco que es una sociedad de economía mixta del orden nacional, del tipo de las anónimas, sujeta al régimen de empresa industrial y comercial del Estado colombiano, es eficiente en los dos años.

4. Dos de los bancos eficientes en 2013, que no lo son en 2014: uno obtuvo solo $72 \%$ de eficiencia y el otro obtuvo $0 \%$.

5. Dos de los bancos eficientes en 2014, que no lo fueron para 2013: muestran eficiencias altas del $95 \%$ y $83 \%$.

6. Tres de las compañías de financiamiento que son eficientes en 2013, para 2014 obtuvieron índice de $0 \%$.

7. Las cooperativas financieras nunca fueron eficientes, incluso sus índices siempre estuvieron por debajo del $50 \%$.

\section{Agradecimientos}

El presente artículo es producto de la investigación Estudio de la eficiencia y la productividad de los sectores de la economía colombiana, financiada por la Universidad Nacional de Colombia, y realizada entre 2015 y hasta agosto de 2016, código interno de registro: 11449 


\section{Referencias}

Allen, F., Demirgüç-Kunt, A., Klapper, L., \& Martínez-Peria, M. S. (2016). The Foundations of Financial Inclusion: Understanding Ownership and Use of Formal Accounts. Journal of Financial Intermediation, 27(C), 1-30.

Alliance for Financial Inclusion, AFI (2011). Maya Declaration. Disponible en: http://www.afi-global.org/publicati ons/879/Maya-Declaration-The-AFI-network-commitment-to-financial-inclusion, http://www.afi-global.org /sites/default/files/publications/2017-04/Maya\%20Declaration_SP.pdf

Alliance for Financial Inclusion, AFI (2013). Putting Financial Inclusion on the Global Map. The 2013 Maya Declaration Progress Report. Bangkok: Alliance for Financial Inclusion. Disponible en: http://www.afi-global.o $\mathrm{rg} /$ publications/1193/The-2013-Maya-Declaration-Report-Putting-Financial-Inclusion-on-the-Global-Map

Alliance for Financial Inclusion, AFI (2016). A Financial Inclusion Index to Measure the Progress of Financial Inclusion. Kuala Lumpur: Alliance for Financial Inclusion. Disponible en: http://www.afi-global.org/sites/default/files/p ublications/2016-08/guidelinenote-18_fidwg_progress_index.pdf

Appleyard, L. (2011). Community Development Finance Institutions (CDFIs): Geographies of Financial Inclusion in the US and UK. Geoforum, 42(2), 250-258.

Banker, R. D., Charnes, A., \& Cooper, W. W. (1984). Some Models for Estimating Technical and Scale Inefficiencies in Data Envelopment Analysis. Management Science, 30(9), 1078-1092.

Banca de las Oportunidades, BdO \& Superintendencia Financiera de Colombia, SFC (2012). Reporte de inclusión financiera 2011. Bogotá: Banca de las Oportunidades, Bancoldex - Banco de Comercio Exterior de Colombia S.A., BdO y Superintendencia Financiera de Colombia, SFC. Disponible en: https://www.superfinanciera.gov. co/descargas?com=institucional\&name $=$ pubFile1000970\&downloadname=18052012reporteinclusion. pdf

Banca de las Oportunidades, BdO \& Superintendencia Financiera de Colombia, SFC (2014). Reporte de inclusión financiera 2013. Bogotá: Banca de las Oportunidades, Bancoldex - Banco de Comercio Exterior de Colombia S.A., BdO y Superintendencia Financiera de Colombia, SFC. Disponible en: http://bancadelasoportunidades. gov.co/sites/default/files/2017-03/reporte_web_0.pdf

Banca de las Oportunidades, BdO \& Superintendencia Financiera de Colombia, SFC (2015). Reporte de inclusión financiera 2014. Bogotá: Banca de las Oportunidades, Bancoldex - Banco de Comercio Exterior de Colombia S.A., BdO y Superintendencia Financiera de Colombia, SFC. Disponible en: http://bancadelasoportunidades.g ov.co/sites/default/files/2017-03/reporte_inclusi\%C3\%B3n_financiera_2014_0.pdf

Bayero, M. A. (2015). Effects of Cashless Economy Policy on Financial Inclusion in Nigeria: An Exploratory Study. Procedia - Social and Behavioral Sciences, 172, 49-56. Disponible en: http://ac.els-cdn.com/S1877042815003717/1-s2.0-S1877042815003717-main.pdf?_tid=a3359f56-4de1 -11e7-b92f-00000aab0f6c\&acdnat=1497101895_e3fa2d6b7eb40e8a8effece455bb5c24

Bhowmik, S. K. \& Saha, D. (2013). Financial Inclusion of the Marginalised Street Vendors in the Urban Economy. New Delhi: Springer.

Center for Financial Inclusion, CFI (2015). Inclusión financiera y envejecimiento: una oportunidad. MetLife Foundation. Disponible en: https://centerforfinancialinclusionblog.files.wordpress.com/2015/05/aging_and_ financial_inclusion_sp.pdf

Cervera-Muñoz, A., Oviedo-García, W., \& Pineda-Acero, J. A. (2013). Revisión bibliográfica de la aplicación de la metodología DEA en el ámbito educativo colombiano. Revista Civilizar Ciencias Sociales y Humanas, 13(25), 133-156. Disponible en: http://www.scielo.org.co/pdf/ccso/v13n25/v13n25a10.pdf

Chakrabarty, K. C. (September 2009). Banking: Key Driver for Inclusive Growth. RBI Monthly Bulletin, 1479-1486. Disponible en: https://rbidocs.rbi.org.in/rdocs/Bulletin/PDFs/4BDIGKCC0909.pdf

Chakravarty, S. R. \& Pal, R. (2013). Financial Inclusion in India: An Axiomatic Approach.Journal of Policy Modeling, 35(5), 813-837.

Charnes, Abraham \& Cooper, William W. (1962). Programming with Linear Fractional Functionals. Naval Research Logistics Quarterly, 9 (3-4), 181-186. 
Chavan, P. (2007). Access to Bank Credit: Implications for Dalit Rural Households. Economic and Political Weekly, 42(31), 3219-3224. Disponible en: https:/questforequity.org/contents/Papers/Access\%20to\%20Bank\%20C redit-\%20Implications\%20for\%20Dalit\%20Rural\%20Households-\%20Pallavi\%20Chavan.pdf

Chediak-Pinzón, F. A. (2008). La técnica DEA para medir y analizar la eficiencia, municipios del departamento del Tolima. Tesis doctoral inédita no publicada, Universidad Tecnológica de Pereira, UTP, Facultad de Ingeniería Industrial, Pereira. Disponible en: http://repositorio.utp.edu.co/dspace/bitstream/handle/11059/1899/6585 036CH514.pdf? sequence $=1$

Coll, V. \& Blasco, O. M. (2006). Evaluación de la eficiencia mediante el análisis envolvente de datos: introducción a los modelos básicos. Madrid: Editorial Eumed. Disponible en: http://www.eumed.net/libros-gratis/2006c/197/

Colombia (2006). Decreto 3078 de 2006 por el cual se crea el Programa de Inversión Banca de las Oportunidades, Diario Oficial, 46.388, 11 de septiembre de 2006. Disponible en: http://www.suin.gov.co/viewDocument.asp ?id=1780311

Colombia (2006). Documento Conpes 3424 de 2006, la banca de las oportunidades, una política para promover el acceso al crédito y a los demás servicios financieros buscando equidad social. Disponible en: http://www.vivase gurofasecolda.com/wp-content/uploads/2016/02/Documento-Conpes-3424-de-2006.pdf

Comisión Multisectorial de Inclusión Financiera, CMIF (2015). Perú: Estrategia Nacional de Inclusión Financiera. Lima: Ministerio de Economía y Finanzas. Disponible en: https://www.mef.gob.pe/contenidos/archivos-desc arga/ENIF.pdf

Committee on Financial Inclusion, CFI (2008). Report of the Committee on Financial Inclusion. New Delhi: Committee on Financial Inclusion, CFI. Disponible en: https://www.sidbi.in/files/Rangarajan-Commitee-rep ort-on-Financial-Inclusion.pdf

Consejo Privado de Competitividad, Compite (2016). Informe Nacional de Competitividad 2014-2015. Bogotá: Consejo Privado de Competitividad, Compite. Disponible en: https://compite.com.co/informe/informe-naci onal-de-competitividad-2014-2015/

Cook, W. D., Kress, M., \& Seiford, L. M. (1993). On the Use of Ordinal Data in Data Envelopment Analysis. Journal of Operational Research Society, JORS, 44(2), 133-140.

Cooper, W. W., Seiford, L. M., \& Tone, K. (2000). Data Envelopment Analysis: A Comprehensive Text with Models, Applications, References and DEA-Solver Software. Boston, Massachusetts: Kluwer Academic Publishers.

D'Alcantara, G. \& Gautier, A. (2013). The Postal Sector as a Vector of Financial Inclusion. Annals of Public and Cooperative Economics, 84 (2), 119-137.

Demirgüç-Kunt, A. \& Klapper, L. (2013). Measuring Financial Inclusion: Explaining Variation in Use of Financial Services Across andwithin Countries. Brookings Papers on Economic Activity, 279-321. Disponible en: https:// www.brookings.edu/wp-content/uploads/2016/07/2013a_klapper.pdf

Diniz, E., Birochi, R., \& Pozzebon, M. (2012). Triggers and Barriers to Financial Inclusion: The Use of ICTBased Branchless Banking in an Amazon County. Electronic Commerce Research and Applications, 11, 484-494. Disponible en: http://gvpesquisa.fgv.br/sites/gvpesquisa.fgv.br/files/arquivos/diniz_-_triggers_and_barriers_t o_financial_inclusion_the_use_of_ict-based_branchless_banking_in_an_amazon_county.pdf

Estados Unidos (1977). Community Reinvestment Act, 12 USC 2901: Title 12-Banks and Banking, Chapter 30, Community Reinvestment. Disponible en: http://uscode.house.gov/view.xhtml?req=granuleid:USC-prelim-ti tle12-section2901\&num=0\&edition=prelim

Fungáčová, Z. \& Weill, L. (2015). Understanding Financial Inclusion in China. China Economic Review, 34, 196-206.

García, N., Grifoni, A., López, J. C., \& Mejía, D. M. (2013). La educación financiera en América Latina y el Caribe. Situación actual y perspectivas. Caracas: Corporación Andina de Fomento, CAF, Banco de Desarrollo de América Latina. Disponible en: https://www.oecd.org/daf/fin/financial-education/OECD_CAF_Financial_Educatio n_Latin_AmericaES.pdf

Ghahraman, A. \& Prior, D. (2016). A Learning Ladder to Ward Efficiency: Proposing Network-Based Stepwise Benchmark Selection. Omega, 63, 83-93. 
Global Financial Development, GFD (2013). Global Financial Development Report: Rethinking the Role of the State in Finance. Washington: International Bank for Reconstruction and Development, The World Bank. Disponible en: https://openknowledge.worldbank.org/bitstream/handle/10986/11848/Global\%20Financial \%20Development\%20Report\%202013.pdf

Global Partnership for Financial Inclusion, GPFI (2016). G20 Financial Inclusion Indicators. Hangzhou, China: GPFI, G 202016 China. Disponible en: https://www.gpfi.org/sites/default/files/Indicators\%20note_formatted.pdf

González-Araya, M. C. \& Valdés, N. G. (2009). Método de selección de variables para mejorar la discriminación en el análisis de eficiencia aplicando modelos DEA. Ingeniería Industrial, 8(2), 45-56. Disponible en: https://dialne t.unirioja.es/descarga/articulo/3998825.pdf

Gupte, R., Venkataramani, B., \& Gupta, D. (2012). Computation of Financial Inclusion Index for India. Procedia - Social and Behavioral Sciences, 37, 133-149. Disponible en: http://ac.els-cdn.com/S1877042812007604/1-s2.0-S1877042812007604-main.pdf?_tid=846e82de-4c8a $-11 \mathrm{e} 7-8 \mathrm{e} 5 \mathrm{c}-00000 \mathrm{aacb362 \& acdnat}=1496954527 \_\mathrm{b} 9 \mathrm{ff} 72 \mathrm{a} 753 \mathrm{c} 091 \mathrm{c} 58117 \mathrm{ed} 5 \mathrm{c} 3131 \mathrm{e} 9 \mathrm{a} 1$

Iyer, K. C. \& Banerjee, P. S. (2016). Measuring and Benchmarking Managerial Efficiency of Project Execution Schedule Performance. International Journal of Project Management, 34(2), 219-236.

Johnes, J., Izzeldin, M., \& Pappas, V. (2014). A Comparison of Performance of Islamic and Conventional Banks 2004-2009. Journal of Economic Behavior \& Organization, 103, 93-107. Disponible en: http://opus.bath.ac.uk /41609/1/JEBO_Johnes_Izzeldin_Pappas_2013.pdf

Johnson, S. \& Arnold, S. (2012). Inclusive Financial Markets: Is Transformation under Way in Kenya? Development Policy Review, 30(6), 719-748.

Kumar, A., Narain, S., \& Rubbani, S. (2015). World Bank Lending for Financial Inclusion: Lessons from Reviews of Select Projects. Washington: Independent Evaluation Group, The World Bank Group. Disponible en: https://openknowledge.worldbank.org/bitstream/handle/10986/21796/949720NWP00PUB0cial0Inclu sion.final.pdf?sequence $=1 \&$ isAllowed $=\mathrm{y}$

Lau, K. H. (2013). Measuring Distribution Efficiency of a Retail Network through Data Envelopment Analysis. International Journal Production Economics, 146(2), 598-611.

Lee, G., Yu, M., \& Wang, -1. (2012). DEA-Based Integrated Relationship of Returns to Scale - An Application to Road Maintenance in Taiwan. Journal of Civil Engineering and Management, 18(5), 709-723.

Lee, K. \& Saen, R. F. (2012). Measuring Corporate Sustainability Management: A Data Envelopment Analysis Approach. International Journal Production Economics, 140(1), 219-226. Disponible en: https://www.researchgate.net/publication/251615210_Measuring_corporate_sustainability_management _A_data_envelopment_analysis_approach

Leeladhar, V. (2006). Taking Banking Services to the Common Man - Financial Inclusion. Reserve Bank of India Bulletin, New Delhi. Disponible en: http://www.bis.org/review/r051214e.pdf

Morgan, P. \& Pontines, V. (2015). Financial Inclusion and Financial Education. En Financial System Stability, Regulation, and Financial Inclusion. Tokyo: Springer.

Pino-Mejías, J. L., Solís-Cabrera, F. M., Delgado-Fernández, M., \& Barea-Barrera, R. C. (2010). Evaluación de la eficiencia de grupos de investigación mediante análisis envolvente de datos (DEA). El profesional de la Información, 19(2), 160-167. Disponible en: https://idus.us.es/xmlui/handle/11441/44842?locale-attribute= en

Pirateque, J. E., Piñeros, J. H., \& Mondragón, L. (2013). Eficiencia en los establecimientos bancarios (EB): una aproximación mediante modelos DEA. Borradores de Economia, 798. Disponible en: http://www.banrep.gov.c o/sites/default/files/publicaciones/archivos/be_798.pdf

Puri, J. \& Yadav, S. P. (2013). A Concept of Fuzzy Input Mix-Efficiency in Fuzzy DEA and its Application. Expert Systems with Applications, 40 (5), 1437-1450.

Puri, J. \& Yadav, S. P. (2014). A Fuzzy DEA Model with Undesirable Fuzzy Outputs and its Application to the Banking Sector in India. Expert Systems with Applications, 41(14), 6419-6432. 
Quintanilha da Silveira, J., Correia Baptista Soares de Mello, J. C., \& Angulo-Meza, L. (2012). Evaluación de la eficiencia de las compañías aéreas brasileñas a través de un modelo híbrido de análisis envolvente de datos (DEA) y programación lineal multiobjetivo. Ingeniare. Revista chilena de ingeniería, 20(3), 331-342. Disponible en: ht tp://www.redalyc.org/articulo.oa?id=77225004007

Rodríguez-Lozano, Gloria Isabel (2011). Indicadores DEA (Data Envelopment Analysis) de eficiencia y productividad para las actividades de extensión universitaria. Aplicación en la Universidad Nacional de Colombia. Bogotá: Centro Editorial, Facultad de Ciencias Económicas, Universidad Nacional de Colombia.

Rodríguez-Lozano, G. I. \& Tarazona-Morales, O. (2015). Unidades de emprendimiento bajo un estudio de eficiencia relativa. Revista Facultad Ciencias Económicas, Universidad Militar Nueva Granada, 23(2), 149-162. Disponible en: https://revistas.unimilitar.edu.co/index.php/rfce/article/view/1613/1377

Sarmiento, M., Cepeda, A., Mutis, H., \& Pérez, J. (2014). Nueva evidencia sobre la eficiencia de la banca colombiana: una medición con modelos de frontera no-paramétricos. Archivos de Economía, Documento 392. Disponible en: https://www.researchgate.net/publication/259384225_Nueva_Evidencia_sobre_la_Eficiencia _de_la_Banca_Colombiana_Una_medicion_con_modelos_de_frontera_no-parametricos

Sen, A. (1999). Development as Freedom. New York: Oxford University Press.

Solo, T. M. (2008). Financial Exclusion in Latin America - Or the Social Costs of Not Banking the Urban Poor. Environment and Urbanization, 20(1), 47-66. Disponible en: http://journals.sagepub.com/doi/pdf/10.1177/ 0956247808089148

Superintendencia de Economía Solidaria, SES (29 de julio de 2016). Superintendencia de Economia Solidaria: Nuestra entidad. Disponible en: http://www.supersolidaria.gov.co/es/nuestra-entidad/objetivos

Superintendencia Financiera de Colombia, SFC (23 de julio de 2016). Acerca de la SFC. Propósito Superior de la Superintendencia Financiera de Colombia. Disponible en: https://www.superfinanciera.gov.co/jsp/loader.jsf?IS ervicio $=$ Publicaciones\&lTipo $=$ publicaciones $\& \mathrm{IFuncion}=\operatorname{loadContenidoPublicacion\& id}=60607$

Svitálková, Z. (2014). Comparison and Evaluation of Bank Efficiency in Selected Countries in EU. Procedia Economics and Finance, 12, 644-653. Disponible en: http://ac.els-cdn.com/S221256711400389X/1-s2.0-S221256711400389X-main.pdf?_tid=1f9d9db2-4d1 c-11e7-a52f-00000aab0f6b\&acdnat $=1497017064$ caa1f401aa052fc6fc473f9102a61205

The Economist Intelligence Unit, EIU (2014). Microscopio Global 2014: Análisis del entorno para la inclusión financiera. New York: EIU. Disponible en: http://scioteca.caf.com/bitstream/handle/123456789/583/eiu_mi crofinance_2014_sp_webr1.pdf?sequence $=1$ \&isAllowed $=\mathrm{y}$

Thorat, U. (2006). Financial Inclusion and Millennium Goals. New Delhi: Reserve Bank of India. Disponible en: ht tp://www.bis.org/review/r060126f.pdf

Toloo, M. (2015). Alternative Minimax Model for Finding the Most Efficient Unit in Data. Computers \& Industrial Engineering, 81(C), 186-194.

Vera, C. \& Titelman, D. (2013). El sistema financiero en América Latina y el Caribe: una caracterización. Santiago de Chile: Comisión Económica para América Latina y el Caribe, CEPAL, Serie Financiamiento para el desarrollo. Disponible en: http://repositorio.cepal.org/bitstream/handle/11362/35880/1/LCL3746_es.pdf

Villasenor, John D.; West, Darrell M. \& Lewis, Robin J. (2015). The 2015 Brookings Financial and Digital Inclusion Project Report. Washington: Center for Technology Innovation at Brookings. Disponible en: https://www.bro okings.edu/wp-content/uploads/2016/06/fdip2015.pdf

Villasenor, John D.; West, Darrell M. \& Lewis, Robin J. (2016). The 2016 Brookings Financial and Digital Inclusion Project Report. Washington: Center for Technology Innovation at Brookings. Disponible en: https://www.bro okings.edu/wp-content/uploads/2016/07/fdip_20160803_project_report.pdf

Wanke, P. \& Barros, C. (2014). Two-Stage DEA: An Application to Major Brazilian Banks. Expert Systems with Applications, 41(5), 2337-2344. Disponible en: http://www.coppead.ufrj.br/upload/publicacoes/ESWA8933 _FINAL.pdf

Wei, Q. \& Yan, H. (2010). A Data Envelopment Analysis (DEA) Evaluation Method Based on Sample Decision Making Units. International Journal of Information Technology \& Decision Making, 9 (4), 601-624. 
World Bank Group (2015). The Little Data Book on Financial Inclusion 2015. Washington: World Bank Group.

Zhou, Z., Sun, L., Yang, W., Liu, W., \& Ma, C. (2013). A Bargaining Game Model for Efficiency Decomposition in the Centralized Model. Computers \& Industrial Engineering, 64(1), 103-108.

\section{Notas}

* Artículo de investigación.

\section{Licencia Creative Commons CC BY 4.0}

Para citar este artículo: Rodríguez-Lozano, Gloria Isabel (2017). Inclusión financiera en Colombia. Análisis de su evolución en términos de eficiencia relativa. Cuadernos de Contabilidad, 18(45), 54-73. https:// doi.org/10.11144/Javeriana.cc 18-45.ifca 